PLANT BIOLOGY

\section{Shoot for the top}

Dev. Cell 38, 186-200 (2016)

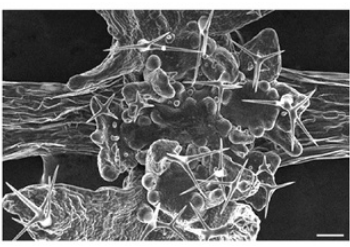

$N^{6}$-Methyladenosine $\left(\mathrm{m}^{6} \mathrm{~A}\right)$ is a posttranscriptional mRNA modification installed by a methyltransferase complex composed of METTL3, METTL14 and WTAP. Although $\mathrm{m}^{6} \mathrm{~A}$ modifications have been observed in a variety of organisms, probing their biological function is challenging because loss-of-function mutations affecting the methyltransferase complexes, for instance in the Arabidopsis WTAP homolog FIP37, often result in embryonic lethality. Shen et al. provided transgenic rescue of FIP37 function to fip 37 mutants during embryonic development and observed that the rescued mutants exhibited an expansion of the shoot apical meristem (SAM), a tissue containing stem cells that generates all aerial organs, with a corresponding loss of leaves and flowers. In situ hybridization analysis revealed that FIP37 was expressed in the SAM and that two SAM regulators, WUSCHEL (WUS) and SHOOTMERISTEMLESS (STM), showed expanded spatial mRNA expression in fip37 mutants. $\mathrm{m}^{6} \mathrm{~A}$ sites were found near the stop codon and 3' UTR in WUS and STM RNA transcripts and were lost in fip37 mutants. The increased STM and WUS RNA expression detected in fip 37 mutants was attributed to increased RNA stability, suggesting that $\mathrm{m}^{6} \mathrm{~A}$ methylation promotes RNA decay and confines WUS and STM mRNA expression to the SAM. Overall, these findings reveal a developmental role of $\mathrm{m}^{6} \mathrm{~A}$ modification in balancing the maintenance and differentiation of a plant stem cell population.

INNATE IMMUNITY

\section{A sweet switch Cell 166, 624-636 (2016)}

Responses to bacterial infections are initiated by pattern recognition receptors (PRRs) of the innate immune system that detect pathogenassociated molecular patterns (PAMPs), molecules produced by degradation of the invading microbe. For example, recent work has shown that degradation of Staphylococcus aureus peptidoglycan (PGN) in macrophages promotes assembly of NOD-like receptor family pyrin domain-containing 3 (NLRP3) inflammasomes that induce secretion of cytokines such as IL-1 $\beta$. Wolf et al. now identify the molecular players in this pathway, showing that $N$-acetylglucosamine (NAG) acts as the PAMP and hexokinase as its PRR. The authors tested possible PGN degradation products and found that cytoplasmic NAG, but not glucosamine (its deacetylated form), led to NLRP3 activation and IL- $1 \beta$ secretion. Because PGN-induced inflammation is thought to involve mitochondria, a search for candidate NAG targets led the authors to hexokinase, a glycolytic enzyme that is localized to the mitochondrial outer membrane and inhibited by NAG. NAG treatment led to hexokinase release into the

\section{GUT MICROBIOME}

\section{Branching into metabolic disease} Nature 535, 376-381(2016)

The composition and metabolic activities of the human gut microbiome can be correlated with metabolic conditions such as insulin resistance (IR). Serum levels of amino acids (particularly branched-chain amino acids, BCAAs), lipopolysaccharides, and certain triacylglycerols and membrane phospholipids have also been linked to IR, raising the possibility that the gut microbiome is a source of these metabolites. To test this idea, Pedersen et al. first determined the fasting serum metabolomes of non-diabetic human patients whose potential for IR or metabolic syndrome was assessed by clinical measures. The metabolome analyses revealed that 19 clusters of metabolites, including BCAAs, lipopolysaccharides and triacylglycerols, were significantly associated with both clinically determined IR and metabolic syndrome (defined as the IR metabotype). The authors then found functional alignments between the metabotype data and microbiome modules in the KEGG database and determined that these correlations are primarily driven by six bacterial species, including Prevotella corpi. Consistent with this finding, individuals with detectable levels of $P$. corpi showed a positive correlation with one BCAA-containing metabolite cluster, and challenging mice fed a high-fat diet with $P$. corpi aggravated insulin resistance and glucose intolerance. These results define an increase in potential BCAA biosynthesis in the gut microbiome of individuals with IR, possibly mediated directly by P. corpi, and a potential causal role of microbially synthesized BCAAs in metabolic disorders. cytoplasm, which was necessary and sufficient for NLRP3 inflammasome formation. Furthermore, treatment of cells with known hexokinase inhibitors or metabolic intermediates that back up the glycolytic pathway also stimulated hexokinase release and inflammasome formation, uncovering a previously unknown link between central metabolism and inflammatory pathways that may have implications in certain metabolic diseases.

CHEMICAL SYNTHESIS

\section{Linking up lysines}

J. Am. Chem. Soc. 138, 8340-8343 (2016)

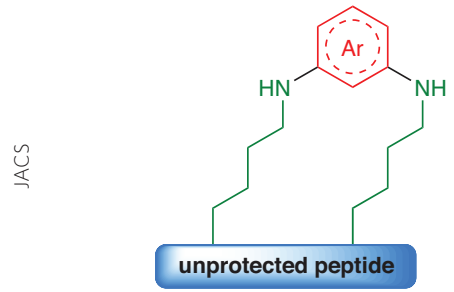

Macrocyclization can endow peptides with enhanced proteolytic stability and cell permeability. Multiple approaches have been developed to generate macrocyclic peptides, including the use of perfluoroaryl compounds that react with cysteine residues to form peptide staples. Lautrette et al. have developed a method that instead uses lysine residues, which yields compounds with improved stability under basic and oxidative conditions compared to compounds produced by the cysteine-based reaction. This method uses an optimized perfluoroaryl reactant and mild conditions to couple two lysine residues via an $\mathrm{S}_{\mathrm{N}}$ Ar mechanism. Peptides containing a range of spacing between the two lysine residues (from adjacent to 14 residues apart) are generally well tolerated by the reaction, which is specific for lysine over other non-cysteine nucleophilic residues. In addition, unnatural lysine analogs with varying side chain lengths can also be stapled. Using this approach, the authors synthesized a macrocyclic analog of a 553 peptide inhibitor of MDM2. This macrocyclic peptide showed better stability toward proteases, increased cell uptake, and improved MDM2 binding compared to the linear peptide. With macrocycles being attractive targets for drug development, the addition of a facile and versatile stapling approach to the peptide toolbox will enable the generation of new macrocyclic peptide scaffolds with the potential for new bioactivities.

Written by Mirella Bucci, Caitlin Deane, Grant Miura \& Terry L. Sheppard 